Proceedings of the 2006 Winter Simulation Conference

L. F. Perrone, F. P. Wieland, J. Liu, B. G. Lawson, D. M. Nicol, and R. M. Fujimoto, eds.

\title{
KNOWLEDGE GLYPHS AS A TACTIC FOR MULTI-PLANAR VISUALIZATION OF SIMULATION PRODUCTS
}

\author{
Gina Thomas-Meyers \\ Human Effectiveness Directorate \\ Air Force Research Laboratory \\ Wright Patterson AFB, OH 45433, U.S.A.
}

\author{
Randall Whitaker \\ Northrop Grumman Information Technology \\ Fairborn, OH 45324, U.S.A.
}

\begin{abstract}
Commanders' Predictive Environment (CPE) is a multiyear program that has been undertaken by the Air Force Research Laboratory to develop ways to allow commanders to better anticipate and predict the outcomes of actions of both allied and opponent forces. One objective of this program is to use intuitive and innovative visualization techniques to present known and alternative courses of action within the command center. One such technique currently under development is termed knowledge glyphs. This paper explains and defines knowledge glyphs and presents initial examples of their use. The current model for knowledge glyphs was developed with the objectives that it be internally coherent, referentially comprehensive, and explicitly linked to the visual interface elements that are most critical to commanders when selecting courses of action.
\end{abstract}

\section{INTRODUCTION}

In the command and control environment, the commander - as ultimate decision maker - will be effective to the extent he or she obtains situation awareness on (a) the current state of the battlespace, (b) prospective courses of action (COA), and (c) the reliability of the information and presumptions supporting the first two items. Currently, decision rationale is typically generated in the form of static presentations (e.g., PowerPoint slides) for the commander's review. Generating these presentations takes significant time - time during which the information they portray may become obsolescent and the most recent intelligence information is lost. The COA options that the commander sees are the options that the intelligence support staff foresaw. The commander needs more direct, dynamic and timely access to information about the battlespace, as well as a means for testing and revising COA parameters in the course of devising a final decision. These information needs require capabilities for richer battlespace visualizations integrated with direct simulation options for the richer visualization elements of those visualizations.
Research jointly undertaken by the Air Force Research Laboratory's (AFRL) Human Effectiveness (HE) and Information (IF) Directorates under the Commanders' Predictive Environment (CPE) program is pursuing such capabilities.

There are two major components involved in the presentation of simulated courses of action: the simulations underlying richer battlespace visualization and innovative interface capabilities for controlling such simulations and assessing their results. The former is the focus of the research being conducted by AFRL/IF and will not be presented in this paper. Rather, we have focused on assisting the human user of the simulation in understanding the implications of both the process and the results of the simulation. This paper is, therefore, focused on visualizations involved in the presentation of simulated information; in particular, we are focused on one dimension of that presentation, the use of symbology. We will define, describe, and exemplify a new category of symbology for the presentation of dynamic information that we refer to as knowledge glyphs.

The notion of a knowledge glyph derives from an understanding of current visualization tools' shortcomings and a desire to create a more sophisticated class of visualization elements. The main thrust of our 2005 work focused on establishing a framework for defining basic constructs and interrelating them with conventional interface or visualization concepts. Currently, we are involved in the design of specific knowledge glyphs to aid in commanders' abilities to anticipate battlespace information. In the following sections, we shall review the issues motivating this work and the results of our most recent efforts and provide examples illustrating how this new class of visual element may be used within a simulation environment.

\section{MOTIVATIONS FOR DEVELOPMENT}

Our top-level objective is to enrich the battlespace visualization tools available to the commander(s). The addition of sophisticated simulation functionalities would permit the commander to evaluate not just the present best-known state of the battlespace, but also alternative states - e.g., 


\section{Thomas-Meyers and Whitaker}

variants on the present state and / or projections based on the present state. For such simulation augmentation to be effectively integrated into next-generation battlespace visualization tools, visualization technologies must accommodate the outboard simulation capabilities. Such accommodation will have to be provided with respect to both (a) triggering simulation support from a given state of a C2 display and (b) coherently portraying the results of said simulation on the same display. In this way, the commander can employ a single battlespace visualization more efficiently (as a user) and more effectively (as a decision maker).

The starting point for our current research was therefore the extent to which current standardized C2 display technologies could support these more sophisticated ends. The US Department of Defense (DoD) has established a standard for symbology to be used in portraying a battlespace. This standard, MIL STD 2525, was deployed in its 'B' revision in 1999 and specifies a structured set of symbology elements which - singly or in integrated combination - provide a basis for illustrating, for example:

- Units,

- Equipment / vehicles,

- Installations,

- A limited set of operations involving the illustrated elements,

- Some limited indication of spatial movement for mobile elements,

- Meteorological and oceanographic (METOC) factors,

- Signals Intelligence (SIGINT) factors, and

- Military Operations Other Than War Symbology (MOOTW) factors.

The MIL STD 2525's symbology set was designed for application in the context of geospatial representations i.e., geographical maps. It is therefore reasonable to characterize MIL STD 2525 as representing to a $21^{\text {st }}$ Century refinement on a Napoleonic era commander's battle map. So long as one assumes a $21^{\text {st }}$ Century commander is adequately supported by a $19^{\text {th }}$ Century information artifact, MIL STD 2525 therefore constitutes something of an advance. Such an assumption is naturally open to question, and it is in the context of questioning this assumption that the standard's limitations become visible.

To illustrate and examine both the limitations of a $19^{\text {th }}$ Century representation and the directions in which constructive improvements lie, we must consider not just the map but also what role the map plays. The classical battle map is a representation of a context (in this case, geospatial) within which a competitive 'game' is being or is to be played out. In this sense, the battle map can be construed as the commander's chessboard. For the sake of illustration, let us continue to consider the metaphor of a chessboard being employed in a game between remotely-located adversaries, neither of whom has direct contact with the other and each of whom must use his or her own chessboard as the sole tool for maintaining situation awareness (SA) about the state of the battle.

There are some ways in which a chessboard continues to be a viable battlespace metaphor in the $21^{\text {st }}$ Century. The chessboard depicts the configuration of a set of elements (pieces) as well as the location of each discrete element. Absent any other information, the chessboard is a static picture of where the pieces are, and nothing more. If one projects upon the chessboard the allowable next moves for each piece, one obtains a static picture of the entire set of next states in the game. If one writes upon each piece data about its basic identity and general capabilities, the set of elements is augmented so as to be self-descriptive upon examination. Figuratively speaking, this metaphor is equivalent to the maximum information one can presume to obtain from a MIL STD 2525 - enabled map.

Although a chessboard may have been a reasonable metaphor for a battlefield in a time of highly-stylized warfare conducted with limited weaponry, it is not a very effective metaphor for modern warfare. The parallelism and complexity of battlespace events are completely different from the linearly stepwise moves of a chess game. Multiple 'pieces' may be moving in unexpected and novel ways - either individually or in concert. Battlespace elements (e.g., vehicles) can shift capabilities from one 'move' to the next. One must always second guess the represented state of the battlespace, because the adversary is capable of masking assets. One must always bear in mind that one or another 'chess piece' may not be there, or may not be what one thinks it is. Moreover, one must also second guess the state of one's own local chessboard, because there is always the possibility that there were errors in assembling the current representation. Finally, states must be considered far in excess of one move ahead in order for effective planning to occur.

All these factors are important in comprehending the adversary's intentions and plans, yet none of these factors are capable of adequate portrayal on our metaphorical chessboard, meaning none of them are capable of adequate portrayal on the equivalent battle map - even with MIL STD 2525 enhancements. Additional symbolic refinements are required to accommodate:

- Uncertainty with regard to the current state of the battlespace.

- Uncertainty with regard to the current state of the battlespace representation itself.

- Uncertainty with regard to the probable future states of the battlespace.

- Richer representation of element dynamics.

- Richer representation of element characteristics. 


\section{Thomas-Meyers and Whitaker}

Generally stated, these factors have provided the motivation for pursuing improved battlespace situation awareness through advanced visualizations and underlie our interest in the development of knowledge glyphs.

In a conventional windowing environment, discrete entities are likely to be graphically portrayed as icons - i.e., graphical units generated in 1-to-1 correspondence to the 'real world' entities they denote in the context of the representation. The features of these icon may be exploited to allow the user to identify what type of entity the icon designates, as is done through shape and other characteristics in MIL STD 2525. However, denoting the entity is often the extent of what an icon does for the user. On a geospatial map, a polyhedron conventionally associated with a tank does no more than indicate that "a tank is located at this set of spatial coordinates." This representation is informative about the tank being situated in the represented geospace, but it tells the user little about the tank itself. Is it active? Is it moving? Is it known to have been destroyed or disabled already? These and other relevant questions cannot be answered by a simple icon - unless, of course, the icon itself is 'loaded up' with additional features or characteristics indicative of such additional information. Therefore, another overriding theme motivating the knowledge glyphs research is a desire to enrich the visualizations provided commanders in the course of planning. In particular, such enrichment needs to be accomplished with respect to the visualization elements used to portray discrete entities within the battlespace representation.

Finally, there are multiple ways in which metainformation such as uncertainty, latency, and pedigree affect the command and control decision making process. Static representations are ill-suited to portray these types of meta-information in a command and control environment As a result, the ability to effectively portray uncertainty and other meta-information has provided motivation for AFRL research on knowledge glyphs.

\section{KNOWLEDGE GLYPH DEVELOPMENT}

Initially, the following conclusions were developed about the task of circumscribing the notion of knowledge glyphs:

- Whatever a 'glyph' may be, it needs to be defined in terms of being a visualization element associated with a given thing or 'entity'.

- The definition must account for the referential context underlying the visualization at hand as well as any additional contexts in which the entity is to be portrayed.

- A 'glyph' needs to be defined as something more than an 'icon.'

- A 'knowledge glyph' must be able to serve as a 'micro-interface' and this function must fit into the otherwise structural definitional framework.
- We need to account for implicit distinctions among 'data', 'information', and 'knowledge' (for our purposes).

These conclusions set the basis for our work. Subsequently, the following points were enumerated as the basis for constructing a working definition of a knowledge glyph:

- A knowledge glyph is a specialized or qualified instance of a glyph.

- A glyph is a visualization element distinct from an 'icon'.

- A glyph is an enhanced version of an icon.

- A glyph can trivially serve as an icon.

- As such, all glyphs are 'iconic', but icons are not necessarily 'glyphic'.

- A knowledge glyph is distinguished from a glyph by virtue of affording users additional leverage on the semantics of the entity being depicted.

- The semantic support afforded by a knowledge glyph lies in its ability to interrelate the denoted entity's projection or occurrence within multiple referential contexts.

In the remainder of this section, we elaborate upon these points and present the first-generation knowledge glyph definition.

\subsection{Icons}

Over the last 20 years we have become accustomed to the term 'icon' denoting a discrete graphical element presented on an interface display. An icon is not just any graphical unit, though some loose usage of the term might lead one to suspect that it can be. Instead, an icon is an icon by virtue of the fact that it denotes an entity of interest in the context of the visual presentation. This characterization of an icon as a form of abstract representation is actually relatively modern, having originated with the semiotic framework of the American philosopher Charles Sanders Peirce. The relevance with respect to Peirce's definition lies in the idea that an icon signifies or designates a single thing in and of itself, 'as is.' An icon does not qualify or describe the entity it signifies; it only denotes it, carrying little or nothing semantic.

The implication of the icon lies not so much in the icon itself as in the referential context in which it is placed. For example, a small dot on a sheet of paper doesn't automatically denote a place where people live. That same dot on a 2-dimensional page of a road atlas can denote a settlement at a particular position in the geographical coordinate system being represented. The dot tells me nothing about the settlement itself. The dot at a particular position in the geo-referenced grid tells me the settlement is 'there'. 


\section{Thomas-Meyers and Whitaker}

For the purposes of this definitional exercise, the term 'icon' is strictly circumscribed to mean no more than what Peirce originally intended. As such, an 'icon' is a display element that serves as no more than a datum within the presentation context. Phrased another way:

An icon is a presentational element which designates an object of reference and specifies its 'locus' in the presentation context.

\subsection{Glyphs}

There are few, if any, clear precedents upon which to base a characterization of a 'glyph' as a distinct class of interface elements. The term 'glyph' has been sporadically applied to a variety of IT-related innovations. However, few of these have to do with interface or visualization issues, and arguably none of these few align with the intent or thrust of the AFRL knowledge glyph research. Examples of such 'glyph' allusions include:

- 'Glyphs' providing programmers a toolkit of simple modular elements for building an interface's graphic structure (Calder and Linton 2003).

- 'Glyphs' as components of a typographical set (Microsoft Typography Standards).

- $\quad$ 'DataGlyphs' as printed digital coding augmenting readable text on document hardcopy (Xerox PARC 2004).

- Pseudo-three-dimensional objects employed for rendering mathematical and other abstract data (e.g., Kindlmann 2004; Chuah and Eick 1998).

Of these examples, it is the last one which comes closest to matching the visualization subject matter at issue here. Even in that case, there is no close correspondence, because 'glyphs' are taken to be visualization data forms whose shape and / or size parameters are exploited to enrich their capacity for portraying complex information. As such, those applications are directed at more sophisticated iconography.

For the purpose of knowledge glyph development, we have deployed another sense of 'glyph' suited to our research purposes. For these purposes, a glyph has been demarcated as an extension to an interface icon. As an icon, a glyph denotes a particular entity of interest. Unlike a basic icon, a glyph affords the user information about that entity above and beyond its presence and its coordinate position in the referential context the visualization represents. More precisely, the first-generation working definition of a glyph is as follows:

A glyph is an icon which additionally affords the user access to information about the denoted entity on its face. This information may be data concerning the de- noted entity or data about available redirection to another presentational device where such additional information may be obtained.

Because the provision of additional information may take either or both of two forms (on the face of the glyph or outboard of the glyph itself), we introduce labeling for these two types of information presentations: intraglyphic and extraglyphic. Intraglyphic presentations are realized on or within the glyph artifact or adjacent to it within the glyph's referential / display context. Extraglyphic presentations are realized on or within a separate referential / display context, which may be implemented as an overlay or other coherent addendum projected onto or otherwise correlated with the glyph's referential / display context.

It is important to note that under this definition an icon capable of being manipulated to invoke outboard data displays qualifies as a glyph if and only if the icon includes some visible cue or mechanism for this extended data access. Lack of a visible cue or mechanism (button, hot spot, etc.) renders the invocation action a feature of the operating system or a subsuming software application - not a feature associated with the form of the visual element (icon) itself.

This visible cueing qualification is not as arbitrary as it may first seem. The point is to define a class of interface artifacts which are clearly distinct from simple icons. This distinction should be evident on the face of the interface artifact itself - even when viewed in isolation from the rest of the on-screen elements. This also relates to the fact that one of the characteristics cited above for knowledge glyphs is their capacity for serving as 'micro-interfaces'. Hidden or secret features are poor interface design practice, and the ascription of fault to such features pertains to 'micro interfaces' as well as to conventional ones.

MIL STD 2525 symbology may be considered a glyph with only intraglyphic information in that it contains information on its face about the entity being represented beyond that of its identity. In Fig. 1, circular means that it represents a surface entity, red means hostile, etc. Extraglyphic information is not available on the face of the MIL STD symbology; as such, it does not address the 'micro-interface' portion of the definition.

\subsection{Knowledge Glyphs}

The final step in defining the knowledge glyph is to specify what qualifies a glyph as a knowledge glyph. For the purposes of the definition, the unique aspect of knowledge glyph has been framed with regard to the mode in which additional information on the denoted entity is afforded the user by virtue of its being a glyph. The provision of no more than descriptive textual data qualifies an icon to be a glyph. To qualify as a knowledge glyph, the visualization element has to be configured so as to provide a vantage on 


\section{Thomas-Meyers and Whitaker}

the denoted entity within a distinct referential context. As a result:

A knowledge glyph is a glyph affording its user the ability to access extraglyphic information in such a form that the extraglyphic presentation is anchored with respect to the same entity (or other discrete object of reference) denoted iconically by the originating glyph.

Under this definition, then, a knowledge glyph implements an intersection among two or more distinct referential contexts in such a manner as to treat the denoted entity as the point of juncture among them all as well as the focal point of reference in the extraglyphic ones. Figuratively speaking, each of the referential contexts is like a 'plane' overlaid on the fundamental coordinate matrix. Because a knowledge glyph depicts a single object or entity across multiple such 'planes,' it in effect affords a foundation for a 'multi-planar' visualization centered on a given object of reference.

Figure 2 illustrates the definitional model for a knowledge glyph. A visual element operates as an icon if it does no more than occupy a meaningful position in the present referential context (e.g., a point on a geospatial grid such as a map). This visual element escalates to the status of a glyph if it affords the viewer additional data either intra- or extraglyphically. The visual element qualifies as a knowledge glyph if and only if it portrays the referenced entity's meaningful position in an alternate referential context (e.g., a timeline rather than a geospatial grid).

\subsection{Illustrative Example}

Let us illustrate the basic utility of a knowledge glyph with an example. Figure 3 illustrates a geospatial map upon which a variety of symbology is overlaid. For the sake of illustration, we shall focus on a single entity on the map - a symbol denoting an adversary armored vehicle. Let us say that we are using an extended version of MIL STD 2525 in which a gold 'cap' is appended to the upper margin of an icon for an entity flagged as mobile or moving. Providing such a 'micro-interface' capability escalates this symbolic device from a simple icon to a glyph.

Now let us say that clicking this glyph's movement indicator triggers a simulation generating the given entity's 'probabilistic movement envelope' - i.e., the geographic area within which the vehicle is capable of relocation within a given period of time. The result of this invoked simulation is illustrated in Figure 4. The shaded 'blob' now overlaid on the original entity depiction represents the probabilistic envelope within which the vehicle might be expected to move or have moved. This envelope is a valueadding projection derived from the entity on the original map, but what is being displayed is not a feature of that en- tity in its original context. In this case, the display has been augmented with visual information coordinated with the geospatial grid of the original map, but projected forward into time (and figuratively into the 'what-if').

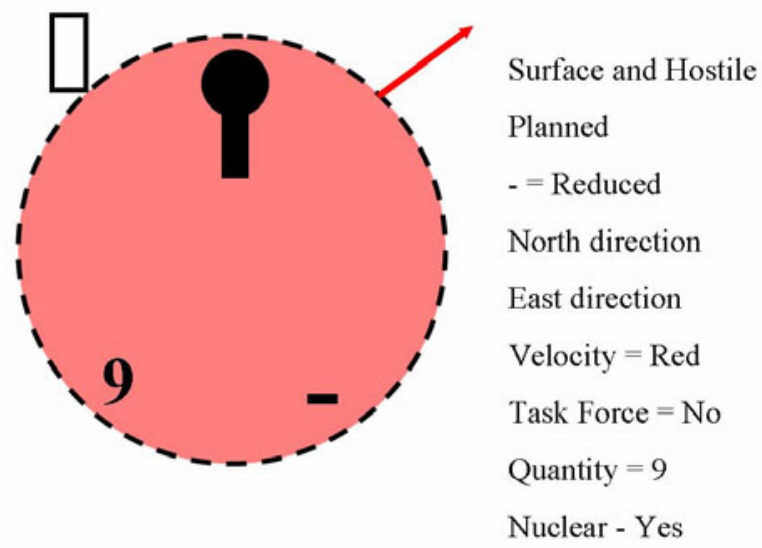

Figure 1: MIL STD 2525 symbol

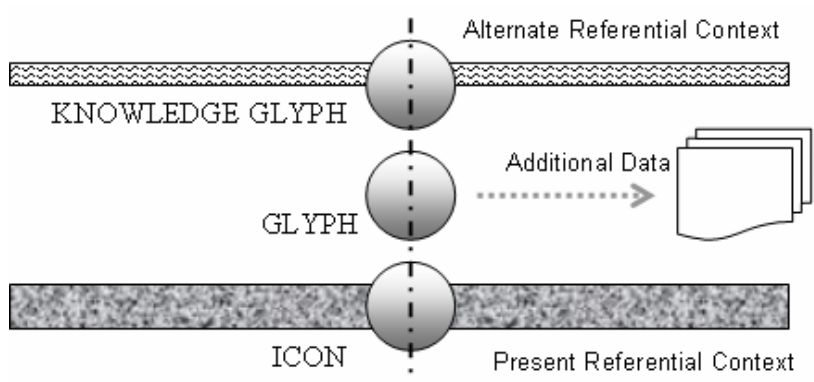

Figure 2: Definitional Model for a Knowledge Glyph

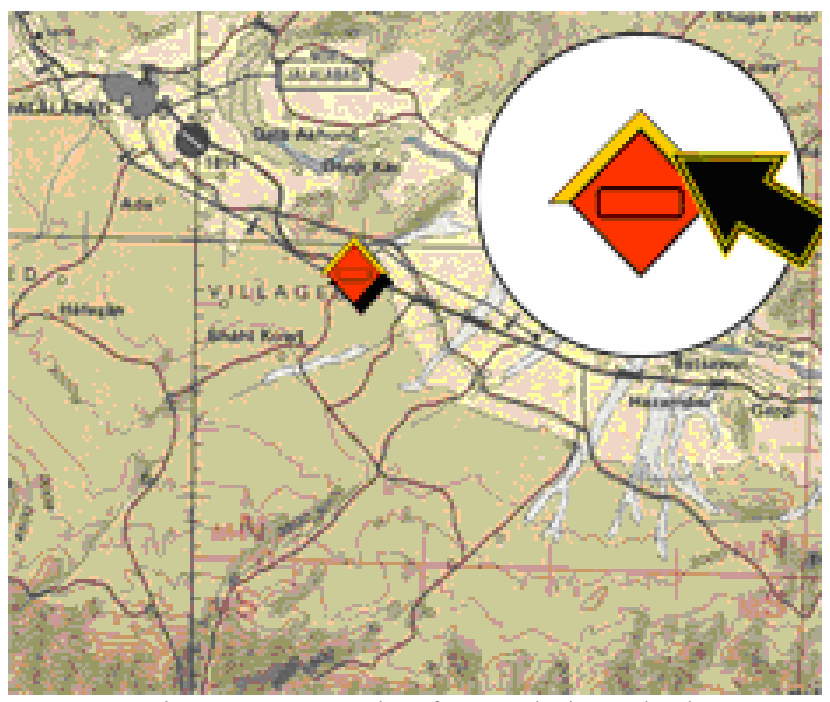

Figure 3: Example of Knowledge Glyph 


\section{Thomas-Meyers and Whitaker}

This new visualization for the entity is portrayed at the same focal point on the display as the original static icon, providing a 'juncture' among multiple referential contexts. The overlay of the entity's probabilistic movement envelope is correlated with the originating position such that the still-visible original icon has significance as an element of both the basic or originating and the overlay representations. Similar such probabilistic representations might be employed to depict characteristics such as estimated ranges of effective fire, distribution of mass assets, and multiple others.

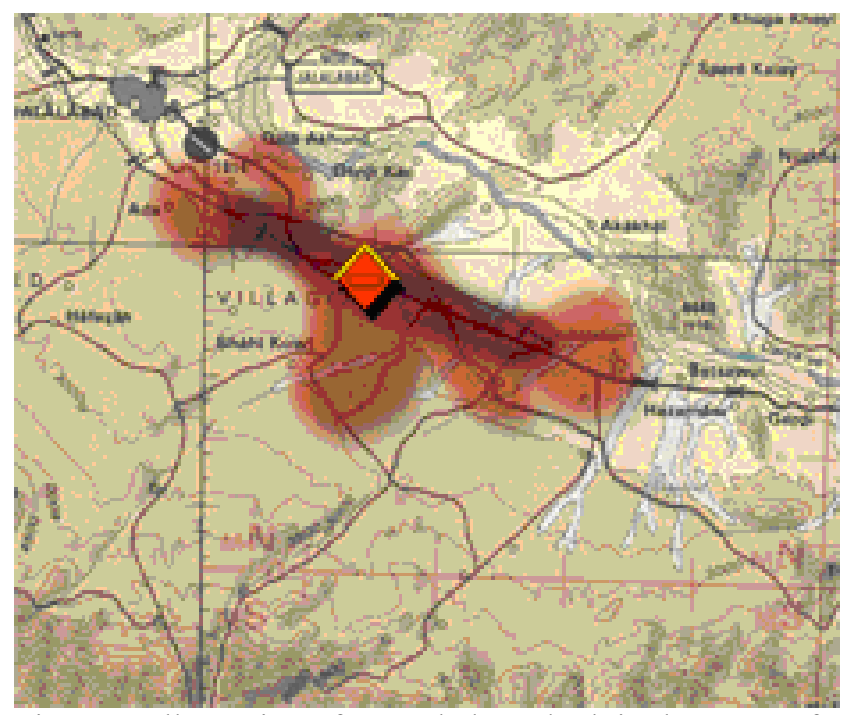

Figure 4: Illustration of Knowledge Glyph in the Form of a Probabilistic Movement Envelope

\section{DISCUSSION}

During the past year and a half, we have undertaken both analytical and theoretical research to further the development of a classification schema for symbology to be employed in Command and Control applications. We have demanded of ourselves that a resultant model be internally coherent, referentially comprehensive, and explicitly linked to the visual interface elements that are not only the foci of our interest but also most relevant to the ability to enhance commanders' predictive capabilities. We have formally defined the intended schema as a hierarchical progression from icons to glyphs to knowledge glyphs.

Establishing this model for integrating and correlating diverse types of symbology in today's software visualizations provides a basis for a number of benefits. First, it affords a useful vocabulary to more critically examine and clearly frame interface visualization specifications as they relate to the target decision maker's focal subject matter. Second, it provides a coherent framework for classifying and evaluating a heretofore unstructured inventory of information visualization tactics. Third, the role of a knowledge glyph as a micro-interface or portal to additional in- formation offers potential users the ability to 'query' or manipulate represented entities individually as they see fit. Fourth, the requirement that a knowledge glyph provide a juncture among multiple referential contexts within one presentational context affords the decision maker the ability to freely examine and explore available data and / or information using a single interface (as opposed to multiple interfaces for each of several 'spins' or 'vantages' on those data or information). Fifth, by virtue of allowing the decision maker to remain referentially and cognitively 'anchored' to a single interface, knowledge glyphs will contribute to more coherent and accurate situation awareness, reduced procedural overhead for information tasks, and reduced risks of confusion and error.

The intrinsic affordances of knowledge glyphs (as 'micro-interfaces') for invoking additional information on their associated battlespace objects provide a means for integrating simulation support into real-time interactions with C2 data. For this purpose, the metaphor of 'microinterface' applies in two directions; the glyph provides a manipulable control interface through which the decision maker may invoke additional information with respect to a selected entity and it provides an anchor for portraying that additional information at the same point of user visual focus at which such an invocation was made.

Our ongoing work is aimed at extending this theoretical foundation toward a new state of the art in visualization techniques and an analogous new state of the art in the usage of these techniques. This model is currently being evaluated in terms of its ability to constructively describe, organize, and explain distinctions among visualization elements. Additionally, a set of sample knowledge glyphs is being designed and constructed. Finally, an ongoing research program is investigating related issues such as the basic perceptual and cognitive mechanisms associated with the portrayal of entities across contexts and their implications for the design of knowledge glyphs for various applications.

\section{ACKNOWLEDGMENTS}

We would like to acknowledge the support of Dr. Michael Young (AFRL/HECS). Key members of an ongoing AFRL/HE Visualization Working Group who have been instrumental to the success of this undertaking include: Dr. Dan Repperger (AFRL/HECP); Ms. Denise Aleva, Dr. Paul Havig, Mr. George Reis, and Ms. Francine Schaffner (AFRL/HECV); Mr. Steve Fullenkamp (General Dynamics); and Mr. Gilbert Kuperman (AFRL/HECS). Additionally, we would like to acknowledge funding support from AFOSR, the Work-Centered Interface Distribution Environment (WIDE) program (managed by Mr. Jeff Wampler, AFRL/HECS), and the Commanders' Predictive Environment (CPE) program (co-managed by Dr. Janet Miller, AFRL/HECS and Jerry Dussault, AFRL/IFS). Current de- 
velopment of knowledge glyphs is being facilitated by the Human Systems Integration Information Analysis Center (HSIIAC).

\section{REFERENCES}

Calder, P. R., and M. A. Linton. 2003. Glyphs: Flyweight Objects for User Interfaces. Stanford University Center for Integrated Systems report available at <http: //www. fresco.org/docs/papers / glyphs.pdf>.

Chuah, M. C., and S. G. Eick. 1998. Information rich glyphs for software management data. IEEE Computer Graphics and Applications, 18(4) July-August, 24-29.

Kindlmann, G. 2004. Superquadric Tensor Glyphs. In Deussen, O.; Hansen, C.; Keim, D.; and D. Saupe. 2004. (Editors), Joint EUROGRAPHICS - IEEE TCVG Symposium on Visualization. Available at <http://www.cs.utah.edu/ gk/papers / vissym0 $4 />$.

Xerox PARC. 2004. Technical Overview of DataGlyphs. Report on research in progress at <http:// www. parc.com/research/projects/datag lyphs/techoverview.html>.

\section{AUTHOR BIOGRAPHIES}

GINA THOMAS-MEYERS is a Research Psychologist with the Air Force Research Laboratory. She previously worked as a Transportation Safety Engineer with the Ohio Department of Transportation. She holds the degrees of Ph.D. and MS in Human Factors Psychology with a focus on vision science as well as a B.S. in Human Factors Engineering, all from Wright State University in Dayton, Ohio. Her research interests include psychophysics, Human Computer Interface, Work-Centered design, and Human Behavioral Representation. She is a member of Vision Science Society, the Optical Society of America, Human Factors and Ergonomics Society, Cognitive Science Society, and Transportation Research Boards. Her e-mail address is <gina. thomas-meyers@wpafb.af.mil>.

RANDALL WHITAKER is a Senior Human Factors Analyst with Northrop Grumman Information Technology. He has received education and training in anthropology / psychology (B.A.), computer science (B.S., M.S.) and informatics (Ph.D.). His research foci have included collaborative IT, cognitive engineering, and advanced interface design. His most recent projects have involved workcentered design of $\mathrm{C} 2$ decision support systems. His e-mail address is <Randall. Whitaker@ngc.com> 